\title{
Smooth trajectory generation and push-recovery based on Divergent Component of Motion
}

\author{
Johannes Englsberger, George Mesesan, Christian Ott
}

\begin{abstract}
This paper presents a novel multi-step closed-form walking trajectory generator based on the concept of Divergent Component of Motion (DCM) that guarantees smoothness of all resulting reference trajectories. Further, we introduce an analytical method for footstep adjustment to recover from strong disturbances. The DCM trajectory is adjusted to guarantee smoothness of control outputs. Additionally, we present a momentum-based disturbance observer that improves robustness w.r.t. strong continuous perturbations. The proposed methods are verified in simulations.
\end{abstract}

\section{INTRODUCTION}

Legged locomotion is generally regarded as a difficult problem due to its hybrid dynamics, unilaterality constraints of contact forces and the high dimensionality and nonlinearity of a robot's general dynamics. For online gait generation, a commonly used idea is to mainly focus on the robot's center of mass (CoM) dynamics, which covers the most important effects of a locomoting system. One of the most popular models following that idea is the Linear Inverted Pendulum (LIP) [1]. Using the LIP dynamics, Tedrake et al. [2] present a closed-form solution for generating walking trajectories from polynomial ZMP references by formulating a linear-quadratic (LQ) problem with the CoM as input. Harada et al. [3] present a method for real-time simultaneous CoM and ZMP planning which allows for smooth gait adjustment. Wieber [4] presents a trajectory-free model predictive controller (MPC), which is based on the minimization of the CoM jerk and can recover from strong perturbations. Recently, the concept of Divergent Component of Motion (DCM) [5], [6] (also known as 'Capture Point' [7]) has become popular in the research community, since it simplifies gait generation and control by only considering the divergent component of the CoM dynamics, while leaving the stable part untouched. Hopkins et al. [8] extended the concept of DCM to a time-varying version.

Gait stabilization can be achieved through three mechanisms: (i) force modulation, as used e.g. in ZMP controllers [2], [6], [8], (ii) modification of angular momentum (e.g. [9], [10]) and (iii) step adjustment (e.g. [5], [10]-[16]). Amongst these methods, step adjustment has the greatest stabilizing potential. Stephens [16] proposes a push recovery method that is based on the generation of full-body step recovery motions. Yun and Goswami [10] present a momentum-based

German Aerospace Center (DLR),

Institute of Robotics and Mechatronics, 82234 Wessling, Germany.

contact: johannes.englsbergerdalr.de reactive stepping controller for level and non-level ground. Urata [11] optimizes three step positions that correspond to stabilizing ZMP trajectories. Takenaka et al. [5] stabilize their walking gait in real-time by enforcing two-step cyclicity of the resulting DCM trajectories via appropriate step adjustment. Khadiv et al. [13] present a DCM-based method for both step location and timing adjustment. The same authors present DCM-based tracking and step adjustment for constant ZMP positions in [14]. Griffin et al. [17] use a DCM-based quadratic program to compute desired ground reaction forces and recovery step locations.

In this paper, we present (i) closed-form solutions for multi-step DCM reference trajectories that are consistent with smooth virtual repellent point (VRP, [6]) trajectories, (ii) an analytical method for step adjustment as well as (iii) a momentum-based disturbance observer. The presented results are purely analytical and achieve a high level of robustness. The paper is structured as follows: Section II presents our method for the generation of smooth and consistent VRP and DCM reference trajectories. Section III introduces an approach for purely analytical footstep adjustment and smooth DCM reference adaptation. Additionally, a momentum-based disturbance observer is presented in Sec. III-C. Sections IV and $\mathrm{V}$ evaluate the proposed methods and conclude the paper.

\section{Generation of CONSISTEnt MUlti-STEP VRP AND DCM REFERENCES}

\section{A. Review of basics on DCM and VRP}

The fundamental theory on Divergent Component of Motion (DCM) and the Virtual Repellent Point (VRP) can be found in our previous work [6], [18]. In this section, we summarize the most important definitions and properties of VRP $\boldsymbol{v}$ and DCM $\boldsymbol{\xi}$. The DCM is defined as

$$
\boldsymbol{\xi}=\boldsymbol{x}+b \dot{\boldsymbol{x}} .
$$

Here, $x$ and $\dot{x}$ denote the center of mass (CoM) position and velocity, respectively, each being a three-dimensional quantity. The DCM time-constant is denoted by $b$, which can be derived from the average height of the CoM above ground surface $\Delta z_{v r p}$ as $b=\sqrt{\Delta z_{v r p} / g}, g$ being the gravitational constant (see [6], [18] for more details).

Reordering (1), we find the CoM dynamics

$$
\dot{\boldsymbol{x}}=-\frac{1}{b}(\boldsymbol{x}-\boldsymbol{\xi}),
$$


which shows that the CoM follows the DCM with a stable first order dynamics. The CoM dynamics can thus (assuming sufficient friction ${ }^{1}$ ) be neglected w.r.t. planning and control, which facilitates the gait design process.

Differentiating (1) and inserting Newton's 2nd law $\ddot{\boldsymbol{x}}=$ $\boldsymbol{F}_{\text {com }} / m$ ( $m$ being the robot's total mass), we find the following unstable first order dynamics for the DCM:

$$
\dot{\xi}=\frac{1}{b}(\boldsymbol{\xi}-\boldsymbol{v})
$$

Here we already inserted the definition of the Virtual Repellent Point (VRP) $v$. The VRP encodes the total force acting on the CoM $\boldsymbol{F}_{\text {com }}$ via

$$
\boldsymbol{F}_{\text {com }}=\frac{m}{b^{2}}(\boldsymbol{x}-\boldsymbol{v}) .
$$

Looking at (3), we find that the DCM is pushed away from the VRP, i.e. it diverges. In the next sections, we will show how this divergent nature of the DCM can be used for the design of contact consistent DCM reference trajectories that obey a certain terminal constraint.

\section{B. Consistent VRP and DCM interpolation as basic element}

In this work, we split the overall preview of future trajectories into a sequence of $n_{\varphi}$ transition phases $\varphi$. During each transition phase $\varphi \in\left\{1, . ., n_{\varphi}\right\}$, we use a polynomial spline to interpolate the VRP reference from the corresponding VRP reference start point $\boldsymbol{v}_{r e f, 0, \varphi} \in \mathbb{R}^{3}$ to the corresponding VRP reference end point $\boldsymbol{v}_{r e f, T, \varphi} \in \mathbb{R}^{3}$. These polynomial splines have the following general form:

$$
\boldsymbol{v}_{r e f, \varphi}\left(t_{\varphi}\right)=\left(1-f_{\varphi}\left(t_{\varphi}\right)\right) \boldsymbol{v}_{r e f, 0, \varphi}+f_{\varphi}\left(t_{\varphi}\right) \boldsymbol{v}_{r e f, T, \varphi} .
$$

where $\boldsymbol{v}_{r e f, 0, \varphi}=\boldsymbol{v}_{r e f, \varphi}(0)$ and $\boldsymbol{v}_{r e f, T, \varphi}=\boldsymbol{v}_{r e f, \varphi}\left(T_{\varphi}\right)$. Here, $t_{\varphi} \in\left[0, T_{\varphi}\right]$ is the local time of transition phase $\varphi$. The transition phase durations $\left(T_{\varphi}\right)_{\varphi=1}^{n_{\varphi}}$ may be predefined or optimized as presented by Mesesan [19]. Table I provides several example functions $f_{\varphi}\left(t_{\varphi}\right)$ together with appropriate boundary conditions for different polynomial orders $n_{\text {poly }}$ that may be used for interpolation. The choice of a particular polynomial order $n_{\text {poly }}$ has an influence on the smoothness of resulting quantities such as VRP, DCM, CoM and joint torques. Thus, this choice depends on the smoothness requirements of the given locomotion task. For increasing polynomial orders, the differentiability of the corresponding VRP reference trajectories increases.

The time-dependent VRP trajectories from (5) can be inserted into the DCM dynamics (3), which results in the following ODE for the DCM reference dynamics

$$
\dot{\boldsymbol{\xi}}_{r e f, \varphi}\left(t_{\varphi}\right)=\frac{1}{b}\left(\boldsymbol{\xi}_{r e f, \varphi}\left(t_{\varphi}\right)-\boldsymbol{v}_{r e f, \varphi}\left(t_{\varphi}\right)\right)
$$

\footnotetext{
${ }^{1}$ If angular momentum is neglected, the desired forces, pointing from the contacting feet towards the robot's CoM, have to lie within the friction cones that correlate to feasible contact forces. Assuming a sufficiently high friction coefficient, the CoM (if above floor level) always lies in the friction cone and can thus be neglected for feasibility considerations.
}

\begin{tabular}{|c|c|l|}
\hline$n_{\text {poly }}$ & $f_{\varphi}\left(t_{\varphi}\right)$ & boundary conditions \\
\hline \hline $0^{\text {th }}$ & 0 & $\boldsymbol{v}_{r e f, \varphi}\left(t_{\varphi}\right)=\boldsymbol{v}_{r e f, 0, \varphi}=$ const. \\
\hline $1^{s t}$ & $\frac{t_{\varphi}}{T_{\varphi}}$ & $\boldsymbol{v}_{r e f, \varphi}(0)=\boldsymbol{v}_{r e f, 0, \varphi}$ \\
& & $\boldsymbol{v}_{r e f, \varphi}\left(T_{\varphi}\right)=\boldsymbol{v}_{r e f, T, \varphi}$ \\
\hline \multirow{3}{*}{$3^{r d}$} & $\left(\frac{t_{\varphi}}{T_{\varphi}}\right)^{2}\left(3-\frac{2 t_{\varphi}}{T_{\varphi}}\right)$ & $\boldsymbol{v}_{r e f, \varphi}(0)=\boldsymbol{v}_{r e f, 0, \varphi}$ \\
& & $\dot{\boldsymbol{v}}_{r e f, \varphi}(0)=0$ \\
& & $\boldsymbol{v}_{r e f, \varphi}\left(T_{\varphi}\right)=\boldsymbol{v}_{r e f, T, \varphi}$ \\
& & $\dot{\boldsymbol{v}}_{r e f, \varphi}\left(T_{\varphi}\right)=0$ \\
\hline \multirow{2}{*}{$5^{t h}$} & $\left.\left(\frac{t_{\varphi}}{T_{\varphi}}\right)^{3}\left(10-\frac{15 t_{\varphi}}{T_{\varphi}}+\frac{6 t_{\varphi}^{2}}{T_{\varphi}^{2}}\right)\right)$ & $\boldsymbol{v}_{r e f, \varphi}(0)=\boldsymbol{v}_{r e f, 0, \varphi}$ \\
& & $\dot{\boldsymbol{v}}_{r e f, \varphi}(0)=0$ \\
& & $\ddot{\boldsymbol{v}}_{r e f, \varphi}(0)=0$ \\
& & $\boldsymbol{v}_{r e f, \varphi}\left(T_{\varphi}\right)=\boldsymbol{v}_{r e f, T, \varphi}$ \\
& & $\dot{\boldsymbol{v}}_{r e f, \varphi}\left(T_{\varphi}\right)=0$ \\
& & $\ddot{\boldsymbol{v}}_{r e f, \varphi}\left(T_{\varphi}\right)=0$ \\
\hline
\end{tabular}

TABLE I

VARIOUS POSSIBLE INTERPOLATION SCHEMES.

Using partial integration, we find the solution to (6) as

$$
\boldsymbol{\xi}_{r e f, \varphi}\left(t_{\varphi}\right)=\boldsymbol{v}_{\Sigma, \varphi}\left(t_{\varphi}\right)+e^{\frac{t \varphi}{b}}\left(\boldsymbol{\xi}_{r e f, 0, \varphi}-\boldsymbol{v}_{\Sigma, 0, \varphi}\right)
$$

where

$$
\boldsymbol{v}_{\Sigma, \varphi}\left(t_{\varphi}\right)=\left(1-\sigma_{\varphi}\left(t_{\varphi}\right)\right) \boldsymbol{v}_{r e f, 0, \varphi}+\sigma_{\varphi}\left(t_{\varphi}\right) \boldsymbol{v}_{r e f, T, \varphi},
$$

with $v_{\Sigma, 0, \varphi}=v_{\Sigma, \varphi}(0)$ and $\sigma_{\varphi}\left(t_{\varphi}\right)=\sum_{j=0}^{n_{p o l y, \varphi}}\left(b^{j} \stackrel{(j)}{f}_{\varphi}\left(t_{\varphi}\right)\right)$, in which $n_{p o l y, \varphi}$ is the polynomial order of $f_{\varphi}\left(t_{\varphi}\right)$ and $\stackrel{(j)}{\square}$ denotes the $j$-th time derivative of any function $\square$. With (8), we reformulate (7) as

$$
\begin{array}{r}
\boldsymbol{\xi}_{r e f, \varphi}\left(t_{\varphi}\right)=\left(1-\sigma_{\varphi}\left(t_{\varphi}\right)-e^{\frac{t \varphi}{b}}\left(1-\sigma_{0, \varphi}\right)\right) \boldsymbol{v}_{r e f, 0, \varphi}+ \\
+\left(\sigma_{\varphi}\left(t_{\varphi}\right)-e^{\frac{t \varphi}{b}} \sigma_{0, \varphi}\right) \boldsymbol{v}_{r e f, T, \varphi}+e^{\frac{t \varphi}{b}} \boldsymbol{\xi}_{r e f, 0, \varphi},
\end{array}
$$

where $\sigma_{0, \varphi}=\sigma_{\varphi}(0)$. This equation returns the reference DCM $\xi_{r e f, \varphi}\left(t_{\varphi}\right)$ at any given time in transition phase $t_{\varphi}$, using the VRP reference start point $\boldsymbol{v}_{r e f, 0, \varphi}$ and end point $\boldsymbol{v}_{r e f, T, \varphi}$ and the DCM reference start point $\boldsymbol{\xi}_{\text {ref, }, \varphi, \varphi}=\boldsymbol{\xi}_{\text {ref, } \varphi}\left(t_{\varphi}=0\right)$ as input. Alternatively, the solution to (6) can be formulated using VRP reference start and end points and the DCM reference end point $\boldsymbol{\xi}_{r e f, T, \varphi}=\boldsymbol{\xi}_{\text {ref, } \varphi}\left(t_{\varphi}=T_{\varphi}\right)$ of each transition phase as boundary condition:

$$
\begin{gathered}
\boldsymbol{\xi}_{r e f, \varphi}\left(t_{\varphi}\right)=\underbrace{\left(1-\sigma_{\varphi}\left(t_{\varphi}\right)-e^{\frac{t \varphi-T_{\varphi}}{b}}\left(1-\sigma_{T, \varphi}\right)\right)}_{\alpha_{\varphi}\left(t_{\varphi}\right)} \boldsymbol{v}_{r e f, 0, \varphi}+ \\
+\underbrace{\left(\sigma_{\varphi}\left(t_{\varphi}\right)-e^{\frac{t \varphi-T_{\varphi}}{b}} \sigma_{T, \varphi}\right)}_{\beta_{\varphi}\left(t_{\varphi}\right)} \boldsymbol{v}_{r e f, T, \varphi}+\underbrace{e^{\frac{t \varphi-T_{\varphi}}{b}}}_{\gamma_{\varphi}\left(t_{\varphi}\right)} \boldsymbol{\xi}_{r e f, T, \varphi} .
\end{gathered}
$$

Summing up: in this section, we have derived consistent VRP and DCM interpolation trajectories, the end results being equations (5) and (10) (or (9), respectively). These quantities 
serve as basic element for designing smooth and consistent multi-step reference trajectories in the following sections.

\section{Computation of multi-step preview matrices}

In this section, we use the consistent VRP and DCM interpolation from above as basic element to interpolate between a sequence of $n_{w p}$ VRP waypoints $\left(\boldsymbol{v}_{w p, j}\right)_{j=1}^{n_{w p}}$ via $n_{\varphi}=n_{w p}-1$ transition phases. During each transition phase $\varphi \in\left\{1, \ldots, n_{\varphi}\right\}$, the corresponding VRP reference trajectory $\boldsymbol{v}_{r e f, \varphi}\left(t_{\varphi}\right)$ is interpolated from the $\varphi$-th VRP waypoint $\boldsymbol{v}_{w p, \varphi}$ to the $(\varphi+1)$-th VRP waypoint $\boldsymbol{v}_{w p, \varphi+1}$, i.e.

$$
\boldsymbol{v}_{r e f, \varphi}\left(t_{\varphi}=0\right)=\boldsymbol{v}_{r e f, 0, \varphi}=\boldsymbol{v}_{w p, \varphi}
$$

and

$$
\boldsymbol{v}_{r e f, \varphi}\left(t_{\varphi}=T_{\varphi}\right)=\boldsymbol{v}_{r e f, T, \varphi}=\boldsymbol{v}_{w p, \varphi+1} .
$$

Collecting all VRP reference start points $\boldsymbol{v}_{r e f, 0, \varphi}$ and waypoints $\boldsymbol{v}_{w p, \varphi}$ in collective vectors $\boldsymbol{v}_{r e f, 0}$ and $\boldsymbol{v}_{w p}$, we find

$$
\underbrace{\left[\begin{array}{c}
\boldsymbol{v}_{r e f, 0,1} \\
\vdots \\
\boldsymbol{v}_{r e f, 0, n_{\varphi}}
\end{array}\right]}_{\boldsymbol{v}_{r e f, 0} \in \mathbb{R}^{3 n \varphi}}=\underbrace{\left[\begin{array}{ccccc}
\boldsymbol{I} & \mathbf{0} & \ldots & \ldots & \mathbf{0} \\
\mathbf{0} & \ddots & \ddots & & \vdots \\
\vdots & \ddots & \ddots & \ddots & \vdots \\
\mathbf{0} & \ldots & \mathbf{0} & \boldsymbol{I} & \mathbf{0}
\end{array}\right]}_{\boldsymbol{A}_{\boldsymbol{v}_{r e f, 0}} \in \mathbb{R}^{3 n \varphi} \times 3 n_{w p}} \underbrace{\left[\begin{array}{c}
\boldsymbol{v}_{w p, 1} \\
\vdots \\
\vdots \\
\boldsymbol{v}_{w p, n_{w p}}
\end{array}\right]}_{\boldsymbol{v}_{w p} \in \mathbb{R}^{3 n_{w p}}}
$$

Similarly, we can also collect all VRP reference end points $\boldsymbol{v}_{r e f, T, \varphi}$ in a collective vector $\boldsymbol{v}_{r e f, T}$

$$
\underbrace{\left[\begin{array}{c}
\boldsymbol{v}_{r e f, T, 1} \\
\vdots \\
\boldsymbol{v}_{r e f, T, n_{\varphi}}
\end{array}\right]}_{\boldsymbol{v}_{r e f, T} \in \mathbb{R}^{3 n \varphi}}=\underbrace{\left[\begin{array}{ccccc}
\mathbf{0} & \boldsymbol{I} & \mathbf{0} & \ldots & \mathbf{0} \\
\vdots & \ddots & \ddots & \ddots & \vdots \\
\vdots & & \ddots & \ddots & \mathbf{0} \\
\mathbf{0} & \ldots & \ldots & \mathbf{0} & \boldsymbol{I}
\end{array}\right]}_{\boldsymbol{A}_{\boldsymbol{v}_{r e f, T}} \in \mathbb{R}^{3 n \varphi \times 3 n_{w p}}} \underbrace{\left[\begin{array}{c}
\boldsymbol{v}_{w p, 1} \\
\vdots \\
\boldsymbol{v}_{w p, n_{w p}}
\end{array}\right]}_{\boldsymbol{v}_{w p} \in \mathbb{R}^{3 n_{w p}}} .
$$

Note: throughout this paper, wherever not stated differently, $\mathbf{0}=\mathbf{0}_{3 \times 3}$ and $\boldsymbol{I}=\boldsymbol{I}_{3 \times 3}$, i.e. typically, three-dimensional quantities are mapped. Now, we derive multi-step DCM reference trajectories that are consistent with the VRP reference trajectories. As in our previous works [6], [18], [20], we use a terminal constraint for the DCM at the end of the preview horizon and use backward iteration to achieve a sequence of $n_{\varphi}$ coherent DCM reference trajectories $\boldsymbol{\xi}_{r e f, \varphi}$. To this end, we evaluate (10) for $t_{\varphi}=0$ to obtain

$$
\begin{gathered}
\boldsymbol{\xi}_{r e f, 0, \varphi}=\underbrace{\left(1-\sigma_{0, \varphi}-e^{-\frac{T_{\varphi}}{b}}\left(1-\sigma_{T, \varphi}\right)\right)}_{\alpha_{0, \varphi}} \boldsymbol{v}_{r e f, 0, \varphi}+ \\
+\underbrace{\left(\sigma_{0, \varphi}-e^{-\frac{T \varphi}{b}} \sigma_{T, \varphi}\right)}_{\beta_{0, \varphi}} \boldsymbol{v}_{r e f, T, \varphi}+\underbrace{e^{-\frac{T_{\varphi}}{b}}}_{\gamma_{0, \varphi}} \boldsymbol{\xi}_{r e f, T, \varphi}
\end{gathered}
$$

which maps each DCM reference end point $\boldsymbol{\xi}_{\text {ref,T, } \varphi}$ and the corresponding VRP reference start point $\boldsymbol{v}_{r e f, 0, \varphi}$ and end point $\boldsymbol{v}_{r e f, T, \varphi}$ to the compatible DCM reference start point $\boldsymbol{\xi}_{r e f, 0, \varphi}$. Using (13) and (14), we write (15) in matrix form

$$
\begin{aligned}
& \underbrace{\left[\begin{array}{c}
\boldsymbol{\xi}_{r e f, 0,1} \\
\vdots \\
\boldsymbol{\xi}_{r e f, 0, n_{\varphi}}
\end{array}\right]}_{\boldsymbol{\xi}_{r e f, 0} \in \mathbb{R}^{3 n \varphi}}=\underbrace{\left[\begin{array}{cccc}
\gamma_{0,1} \boldsymbol{I} & \mathbf{0} & \ldots & \mathbf{0} \\
\mathbf{0} & \ddots & \ddots & \vdots \\
\vdots & \ddots & \ddots & \mathbf{0} \\
\mathbf{0} & \cdots & \mathbf{0} & \gamma_{0, n_{\varphi}} \boldsymbol{I}
\end{array}\right]}_{\boldsymbol{A}_{\gamma} \in \mathbb{R}^{3 n \varphi \times 3 n \varphi}} \underbrace{\left[\begin{array}{c}
\boldsymbol{\xi}_{r e f, T, 1} \\
\vdots \\
\boldsymbol{\xi}_{r e f, T, n_{\varphi}}
\end{array}\right]}_{\boldsymbol{\xi}_{r e f, T} \in \mathbb{R}^{3 n \varphi}}+ \\
& +\underbrace{\left[\begin{array}{ccccc}
\alpha_{0,1} \boldsymbol{I} & \beta_{0,1} \boldsymbol{I} & \mathbf{0} & \ldots & \mathbf{0} \\
\mathbf{0} & \ddots & \ddots & \ddots & \vdots \\
\vdots & \ddots & \ddots & \ddots & \mathbf{0} \\
\mathbf{0} & \cdots & \mathbf{0} & \alpha_{0, n_{\varphi}} \boldsymbol{I} & \beta_{0, n_{\varphi}} \boldsymbol{I}
\end{array}\right]}_{\boldsymbol{A}_{\alpha \beta} \in \mathbb{R}^{3 n \varphi \times 3 n_{w p}}} \underbrace{\left[\begin{array}{c}
\boldsymbol{v}_{w p, 1} \\
\vdots \\
\vdots \\
\boldsymbol{v}_{w p, n_{w p}}
\end{array}\right]}_{\boldsymbol{v}_{w p}}
\end{aligned}
$$

For the generation of consistent VRP and DCM reference trajectories, we use the terminal constraint that the reference DCM comes to a stop, i.e. $\dot{\boldsymbol{\xi}}_{\text {ref }}\left(t_{n_{\varphi}}=T_{n_{\varphi}}\right)=0$ (see (3)) at the end of the complete preview horizon, corresponding to

$$
\boldsymbol{\xi}_{r e f, T, n_{\varphi}}=\boldsymbol{v}_{w p, n_{w p}}
$$

Starting from this terminal constraint, we design the DCM reference end points $\boldsymbol{\xi}_{\text {ref,T, } \varphi}$ of each transition phase $\varphi \in$ $\left\{1, . ., n_{\varphi}-1\right\}$ to coincide with the DCM reference start points $\boldsymbol{\xi}_{\text {ref }, 0, \varphi+1}$ of each subsequent transition phase, i.e.

$$
\xi_{\text {ref }, T, \varphi}=\xi_{\text {ref }, 0, \varphi+1} \quad \forall \varphi \in\left\{1, . ., n_{\varphi}-1\right\} .
$$

This backward iteration scheme assures continuity from one transition phase to the next.

Equations (17) and (18) can be summarized in matrix form

$$
\begin{aligned}
& \underbrace{\left[\begin{array}{c}
\boldsymbol{\xi}_{r e f, T, 1} \\
\vdots \\
\boldsymbol{\xi}_{r e f, T, n_{\varphi}}
\end{array}\right]}_{\boldsymbol{\xi}_{r e f, T}}=\underbrace{\left[\begin{array}{ccccc}
\mathbf{0} & \boldsymbol{I} & \mathbf{0} & \ldots & \mathbf{0} \\
\vdots & \ddots & \ddots & \ddots & \vdots \\
\vdots & & \ddots & \ddots & \mathbf{0} \\
\vdots & & & \ddots & \boldsymbol{I} \\
\mathbf{0} & \ldots & \ldots & \ldots & \mathbf{0}
\end{array}\right]}_{\boldsymbol{A}_{B W I} \in \mathbb{R}^{3 n \varphi \times 3 n \varphi}} \underbrace{\left[\begin{array}{c}
\boldsymbol{\xi}_{r e f, 0,1} \\
\vdots \\
\vdots \\
\boldsymbol{\xi}_{r e f, 0, n_{\varphi}}
\end{array}\right]}_{\boldsymbol{\xi}_{r e f, 0}} \\
& +\underbrace{\left[\begin{array}{ccccc}
\mathbf{0} & \ldots & \ldots & \ldots & \mathbf{0} \\
\vdots & \ddots & & & \vdots \\
\vdots & & \ddots & & \vdots \\
\vdots & & & \mathbf{0} & \mathbf{0} \\
\mathbf{0} & \ldots & \ldots & \mathbf{0} & \boldsymbol{I}
\end{array}\right]}_{\boldsymbol{A}_{T C} \in \mathbb{R}^{3 n \varphi \times 3 n_{w p}}} \underbrace{\left[\begin{array}{c}
\boldsymbol{v}_{w p, 1} \\
\vdots \\
\vdots \\
\boldsymbol{v}_{w p, n_{w p}}
\end{array}\right]}_{\boldsymbol{v}_{w p}}
\end{aligned}
$$

The subscripts "BWI" and "TC" in matrices $\boldsymbol{A}_{B W I}$ and $\boldsymbol{A}_{T C}$ stand for "backward iteration" and "terminal constraint", respectively. Inserting $\boldsymbol{\xi}_{\text {ref,T }}$ from (19) into (16) and solving 
for the DCM reference start point vector $\boldsymbol{\xi}_{r e f, 0}$ we find

$$
\boldsymbol{\xi}_{r e f, 0}=\underbrace{\left(\boldsymbol{I}_{3 n_{\varphi} \times 3 n_{\varphi}}-\boldsymbol{A}_{\gamma} \boldsymbol{A}_{B W I}\right)^{-1}\left(\boldsymbol{A}_{\alpha \beta}+\boldsymbol{A}_{\gamma} \boldsymbol{A}_{T C}\right)}_{\boldsymbol{A}_{\boldsymbol{\xi}_{r e f}, 0} \in \mathbb{R}^{3 n \varphi \times 3 n_{w p}}} \boldsymbol{v}_{w p} \text {. }
$$

Alternatively, inserting $\boldsymbol{\xi}_{\text {ref }, 0}$ from (16) into (19) yields the DCM reference end point vector $\boldsymbol{\xi}_{\text {ref }, T}$ as

$$
\boldsymbol{\xi}_{\text {ref }, T}=\underbrace{\left(\boldsymbol{I}_{3 n_{\varphi} \times 3 n_{\varphi}}-\boldsymbol{A}_{B W I} \boldsymbol{A}_{\gamma}\right)^{-1}\left(\boldsymbol{A}_{T C}+\boldsymbol{A}_{B W I} \boldsymbol{A}_{\alpha \beta}\right)}_{\boldsymbol{A}_{\xi_{\text {ref }, T}} \in \mathbb{R}^{3 n \varphi \times 3 n_{w p}}} \boldsymbol{v}_{w p} .
$$

The inverted matrices in (20) and (21) are invertible for all $T_{\varphi}>0$. As final contribution of this section, we rewrite (10) to directly relate the VRP waypoint vector $\boldsymbol{v}_{w p}$ to the DCM reference $\boldsymbol{\xi}_{\text {ref }, \varphi}\left(t_{\varphi}\right)$ given the transition phase $\varphi$ and time $t_{\varphi}$

$$
\boldsymbol{\xi}_{r e f, \varphi}\left(t_{\varphi}\right)=\boldsymbol{A}_{\boldsymbol{\xi}_{r e f}, \varphi}\left(t_{\varphi}\right) \boldsymbol{v}_{w p} .
$$

The matrix $\boldsymbol{A}_{\xi_{\text {ref }}, \varphi}\left(t_{\varphi}\right)$ can be computed as

$$
\begin{aligned}
\boldsymbol{A}_{\boldsymbol{\xi}_{\text {ref },}, \varphi}\left(t_{\varphi}\right) & =\alpha_{\varphi}\left(t_{\varphi}\right) 3 \operatorname{row}\left(\boldsymbol{A}_{\left.\boldsymbol{v}_{\text {ref }, 0}, \varphi\right)}\right. \\
& +\beta_{\varphi}\left(t_{\varphi}\right) 3 \operatorname{row}\left(\boldsymbol{A}_{\left.\boldsymbol{v}_{\text {ref }, T}, \varphi\right)}\right. \\
& +\gamma_{\varphi}\left(t_{\varphi}\right) 3 \operatorname{row}\left(\boldsymbol{A}_{\left.\boldsymbol{\xi}_{\text {ref }, T}, \varphi\right) .}\right.
\end{aligned}
$$

Here we introduce the following definition:

\section{Definition 1:}

The operator $3 \operatorname{row}(\boldsymbol{M}, i)$ selects a block of three rows from the $(3 i-2)$-th to the $(3 i)$-th row of matrix $M$.

Summing up: in this section we found equations (13), (14), (20) and (21) which map an arbitrary VRP waypoint vector $\boldsymbol{v}_{w p}$ to the corresponding VRP reference start point vector $\boldsymbol{v}_{r e f, 0}$, VRP reference end point vector $\boldsymbol{v}_{r e f, T}$, DCM reference start point vector $\boldsymbol{\xi}_{\text {ref }, 0}$ and DCM reference end point vector $\boldsymbol{\xi}_{\text {ref }, T}$, respectively. Finally, we found equation (22), which provides a very compact mapping from the VRP waypoint vector $\boldsymbol{v}_{w p}$ to the DCM reference position $\boldsymbol{\xi}_{r e f, \varphi}\left(t_{\varphi}\right)$ for a given time $t_{\varphi}$ in transition phase $\varphi$.

\section{Ensuring continuity for standing-to-walking transitions}

In our framework, when the robot is standing, the VRP reference position $\boldsymbol{v}_{\text {refstand }}$ and DCM reference position $\xi_{\text {ref,stand }}$ coincide, such that the DCM reference velocity $\dot{\xi}_{\text {ref,stand }}$ is zero (stationary case, see (3)). As the title of this paper indicates, we are looking for smooth trajectory generation, which includes smooth transitions from standing to walking and finally back to standing. Looking at the DCM control law presented in [6], [18], [20]

$$
\boldsymbol{v}=\boldsymbol{v}_{r e f, \varphi}\left(t_{\varphi}\right)+\left(\boldsymbol{I}+\boldsymbol{K}_{\boldsymbol{\xi}} b\right)\left(\boldsymbol{\xi}-\boldsymbol{\xi}_{r e f, \varphi}\left(t_{\varphi}\right)\right),
$$

we find that at the transitions from standing to walking and back (as well as throughout the gait sequence) both the VRP and the DCM reference should be continuous in order to avoid discontinuities in the controlled VRP $v$ trajectory and the corresponding desired forces on the CoM, and thus joint torques. To this end, we make following design choices with regard to the VRP waypoints of the walking sequence:

- D1: The first VRP waypoint $\boldsymbol{v}_{w p, 1}$ coincides with the initial standing VRP reference, i.e. $\boldsymbol{v}_{w p, 1}=\boldsymbol{v}_{\text {ref,stand,ini }}$.

- D2: The first DCM reference start point $\xi_{r e f, 0,1}$ also coincides with the initial standing VRP reference point, i.e. $\boldsymbol{\xi}_{\text {ref }, 0,1}=\boldsymbol{v}_{\text {ref,stand,ini }}$.

- D3: The final VRP waypoint $\boldsymbol{v}_{w p, n_{w p}}$ coincides with the final standing VRP reference, i.e. $\boldsymbol{v}_{w p, n_{w p}}=\boldsymbol{v}_{\text {ref,stand,end }}$.

Note that the terminal DCM reference end point $\boldsymbol{\xi}_{r e f, T, n_{\varphi}}$ is already constrained to coincide with the final VRP waypoint $\boldsymbol{v}_{w p, n_{w p}}$ via the terminal constraint (17) and thus with the final standing VRP reference point $\boldsymbol{v}_{\text {ref,stand,end }}$ via D3, such that no additional design choice/constraint has to be introduced here. The constraints introduced by our design choices D1 to D3 can be expressed in matrix form:

$$
\underbrace{\left[\begin{array}{c}
\boldsymbol{v}_{\text {ref,stand,ini }} \\
\boldsymbol{v}_{\text {ref,stand,ini }} \\
\boldsymbol{v}_{\text {wp }, u c} \\
\boldsymbol{v}_{r e f, s t a n d, \text { end }}
\end{array}\right]}_{\boldsymbol{b}_{w p} \in \mathbb{R}^{3 n_{w p}}}=\underbrace{\left[\begin{array}{cccc}
\boldsymbol{I} & \mathbf{0} & \ldots & \mathbf{0} \\
& 3 \operatorname{row}\left(\boldsymbol{A}_{\boldsymbol{\xi}_{\text {ref }, 0}}, 1\right) \\
\mathbf{0}_{n_{u c}} & \mathbf{0}_{n_{u c}} & \boldsymbol{I}_{n_{u c}} & \mathbf{0}_{n_{u c}} \\
\mathbf{0} & \cdots & \mathbf{0} & \boldsymbol{I}
\end{array}\right]}_{\boldsymbol{B}_{w p} \in \mathbb{R}^{3 n_{w p} \times 3 n_{w p}}} \boldsymbol{v}_{w p} .
$$

Here, $\boldsymbol{b}_{w p}$ denotes the VRP waypoint boundary condition vector and $\boldsymbol{B}_{w p}$ is the VRP waypoint boundary condition matrix, with $\mathbf{0}_{n_{u c}} \in \mathbb{R}^{n_{u c} \times 3}$ and $\boldsymbol{I}_{n_{u c}} \in \mathbb{R}^{n_{u c} \times n_{u c}}$. The design choices D1 to D3 are implemented via the first, second and fourth row of (25), respectively. The $n_{u c}=n_{w p}-3$ remaining degrees of freedom ("uc" stands for "unconstrained") are expressed by the collective vector of unconstrained VRP waypoints $\boldsymbol{v}_{w p, u c} \in \mathbb{R}^{n_{u c}}$, which can be freely assigned by a controls engineer or planning algorithm. The second row of (25) is the most peculiar one. It encodes the design choice D2 as $\boldsymbol{\xi}_{\text {ref }, 0,1}=3 \operatorname{row}\left(\boldsymbol{A}_{\left.\boldsymbol{\xi}_{\text {ref }, 0}, 1\right)} \boldsymbol{v}_{w p}=\boldsymbol{v}_{\text {ref,stand,ini. }}\right.$. A close look at the structure of $\boldsymbol{B}_{w p}$ reveals that design choice D2 is implemented through the second VRP waypoint $\boldsymbol{v}_{w p, 2}$. Inversion of (25) yields

$$
\boldsymbol{v}_{w p}=\boldsymbol{B}_{w p}^{-1} \boldsymbol{b}_{w p},
$$

which maps the VRP waypoint boundary condition vector $\boldsymbol{b}_{w p}$ to a VRP waypoint vector $\boldsymbol{v}_{w p}$ that is compatible with design choices D1 to D3 and thus fulfills our continuity requirements. Note: matrix $\boldsymbol{B}_{w p}$ is invertible for all $T_{\varphi}>0$.

\section{Push Recovery Via Step adjustment}

In the previous sections, we derived analytical expressions for all relevant VRP and DCM reference quantities which correlate to a multi-step preview. The resulting matrix mappings will now be used to derive an adequate footstep adjustment in Sec. III-A and an adjustment of the DCM reference trajectory in Sec. III-B, which ensures smoothness of all resulting trajectories. The combination of these two methods allows for smooth recovery from strong and even persistent unknown perturbations. Note that they work together (rather than being alternatives) to accomplish the push recovery task. 


\section{A. Analytical computation of step adjustment}

In this section, we derive an algorithm for step adjustment, which allows for the recovery from strong pushes. One feature of the presented step adjustment is that it correlates to smooth VRP transitions/trajectories ${ }^{2}$, just like in the nominal case presented above. A key feature of the overall method presented in this paper is the linearity of most presented quantities such as matrix mappings. One such linear quantity is given by equation (20). In case of an adjustment of the VRP waypoint vector $\boldsymbol{v}_{w p}$ by $\Delta \boldsymbol{v}_{w p}$, the same matrix $\boldsymbol{A}_{\boldsymbol{\xi}_{\text {ref }, 0}}$ can be applied to determine the corresponding effect on the DCM reference start point vector:

$$
\underbrace{\boldsymbol{\xi}_{a d j, 0}}_{=\boldsymbol{\xi}_{r e f, 0}+\Delta \boldsymbol{\xi}_{a d j, 0}}=\boldsymbol{A}_{\boldsymbol{\xi}_{\text {ref }, 0}} \underbrace{\boldsymbol{v}_{w p, a d j}}_{\boldsymbol{v}_{w p}+\Delta \boldsymbol{v}_{w p}} .
$$

Here, the index "adj" denotes adjusted quantities, while "ref" denotes nominal reference quantities as derived in the previous sections. This shows that a VRP waypoint vector adjustment $\Delta \boldsymbol{v}_{w p}$ can be directly related to the corresponding difference in the DCM reference start point vector $\Delta \boldsymbol{\xi}_{a d j, 0}$ via

$$
\Delta \boldsymbol{\xi}_{a d j, 0}=\boldsymbol{A}_{\boldsymbol{\xi}_{r e f, 0}} \Delta \boldsymbol{v}_{w p} .
$$

Remember that the VRP waypoint vector $\boldsymbol{v}_{w p}$ forms a collection of all VRP waypoints. Similarly, the VRP adjustment vector is $\Delta \boldsymbol{v}_{w p}=\left[\Delta \boldsymbol{v}_{w p, 1}^{T}, \ldots, \Delta \boldsymbol{v}_{w p, n_{w p}}^{T}\right]^{T}$. In future extensions of this work, each element of this VRP adjustment vector could be used independently to achieve certain goals, which might for example be formulated as an optimization problem. In this paper, we choose a more particular form:

$$
\Delta \boldsymbol{v}_{w p}=\underbrace{[\mathbf{0}, \ldots, \mathbf{0}, \boldsymbol{I}, \ldots, \boldsymbol{I}, \mathbf{0}, \ldots, \mathbf{0}]^{T}}_{\boldsymbol{S}_{a d j} \in \mathbb{R}^{3 n_{w p} \times 3}} \Delta \boldsymbol{v}_{a d j} .
$$

Here, $\boldsymbol{S}_{a d j}$ denotes the adjustment selection matrix and $\Delta \boldsymbol{v}_{\text {adj }} \in \mathbb{R}^{3}$ is the VRP adjustment that is equally applied to all modifiable VRP waypoints. The latter are all VRP waypoints that correspond to the next modifiable footstep. The extension to several different footstep and thus VRP waypoint adjustments is beyond the scope of this paper.

In the following, we will denote the transition phase, in which the corresponding VRP interpolation is first affected by the VRP adjustment (compare to matrix $\boldsymbol{S}_{a d j}$ in (29)), as $\varphi_{f a}$, while the index of the first adjusted VRP waypoint is denoted by $i_{f a, w p}$. The correlation between these two quantities is $\varphi_{f a}=i_{f a, w p}-1$. Applying the 3row operator as defined above to (28) and inserting (29) we find

$$
\Delta \boldsymbol{\xi}_{a d j, 0, \varphi_{f a}}=3 \operatorname{row}\left(\boldsymbol{A}_{\boldsymbol{\xi}_{\text {ref }, 0}}, \varphi_{f a}\right) \underbrace{\boldsymbol{S}_{a d j} \Delta \boldsymbol{v}_{a d j}}_{\Delta \boldsymbol{v}_{w p}}
$$

\footnotetext{
${ }^{2}$ Note: smoothness is only guaranteed for the adjusted VRP reference trajectories, while the corresponding adjusted DCM reference trajectories are smooth if and only if the disturbances are continuous, i.e. the measured/estimated DCM don't jump, which would correspond to a Dirac impulse (e.g. hit with a hammer).
}

The 3row operator selects the three rows of matrix $\boldsymbol{A}_{\boldsymbol{\xi}_{\text {ref } .0}}$ that map the VRP adjustment vector $\Delta \boldsymbol{v}_{w p}$ to the $\varphi_{f a}$-th DCM reference start point adjustment $\Delta \boldsymbol{\xi}_{a d j, 0, \varphi_{f a}}$. Here, we assume that the currently ${ }^{3}$ measured DCM tracking error ${ }^{4} \tilde{\xi}=\boldsymbol{\xi}-$ $\boldsymbol{\xi}_{\text {ref, } \varphi}\left(t_{\varphi}\right)$ persists until the instant of step adjustment, i.e.

$$
\Delta \boldsymbol{\xi}_{a d j, 0, \varphi_{f a}}=\tilde{\xi}
$$

Other assumptions such as asymptotic convergence due to the controller action or divergence of the DCM due to actuation constraints are conceivable as well. Yet, in the context of perturbations, these other assumptions have the disadvantages of idleness and over-aggressiveness, respectively, as compared to the assumption chosen here. Inserting (31) into (30) and solving yields the sought-after step adjustment

$$
\Delta \boldsymbol{v}_{a d j}=\underbrace{\left(3 \operatorname{row}\left(\boldsymbol{A}_{\boldsymbol{\xi}_{\text {ref }, 0},} \varphi_{f a}\right) \boldsymbol{S}_{a d j}\right)^{-1}}_{\boldsymbol{A}_{a d j} \in \mathbb{R}^{3 \times 3}} \tilde{\boldsymbol{\xi}} .
$$

The matrix $\boldsymbol{A}_{a d j}$ directly maps the current DCM error $\tilde{\boldsymbol{\xi}}$ to an appropriate step/VRP waypoint adjustment $\Delta \boldsymbol{v}_{a d j}$ that (in case of no further perturbations) leads to cancellation of the DCM error at the instant of step adjustment and routes back of the adjusted DCM reference trajectory to the original one via smoothly adjusted VRP trajectories. Note: $\boldsymbol{A}_{a d j}$ is invertible if all $T_{\varphi}>0$ and $S_{a d j}$ is full rank.

Note: In general, the resulting adjusted eCMPs (see [6]) should lie on the ground surface to assure good feasibility (i.e. force focusing) of ground reaction forces. As an example: in the walking simulations presented in Sec. IV only the horizontal components of the nominal 3D VRP waypoint adjustment $\Delta \boldsymbol{v}_{a d j}$ as computed in (32) are used, since the ground that the robot is walking on is flat.

\section{B. Smooth adaptation of DCM reference}

The step adjustment method presented in the previous section returns a discrete information about the location where to step to recover from strong perturbations. In order to avoid discontinuities in the commanded control outputs, in this section we present a method that guarantees smoothness via the tracking of a continuously adjusted DCM reference trajectory. It will be shown that stability is achieved by a combination of DCM reference tracking and step adjustment.

In the left underbrace of equation (27), we already defined the adjusted DCM reference start point vector as $\boldsymbol{\xi}_{\text {adj }, 0}=$ $\boldsymbol{\xi}_{r e f, 0}+\Delta \boldsymbol{\xi}_{a d j, 0}$. Equivalently, for the current transition phase $\varphi$ and the corresponding time in transition $t_{\varphi}$, we find the adjusted DCM reference position $\boldsymbol{\xi}_{a d j, \varphi}\left(t_{\varphi}\right)$ as

$$
\boldsymbol{\xi}_{a d j, \varphi}\left(t_{\varphi}\right)=\boldsymbol{\xi}_{r e f, \varphi}\left(t_{\varphi}\right)+\Delta \boldsymbol{\xi}_{a d j, \varphi}\left(t_{\varphi}\right) .
$$

Here, $\boldsymbol{\xi}_{r e f, \varphi}\left(t_{\varphi}\right)$ is the nominal DCM reference position from (22). Using matrix $\boldsymbol{A}_{\boldsymbol{\xi}_{\text {ref }}, \varphi}\left(t_{\varphi}\right)$ as defined in (23), the DCM

\footnotetext{
${ }^{3}$ Note: in this section, the index $\varphi$ denotes the current transition phase ${ }^{4}$ operator $\tilde{\square}$ stands for tracking errors, the $\Delta \square$ operator for adjustments.
} 
reference adjustment $\Delta \boldsymbol{\xi}_{a d j, \varphi}\left(t_{\varphi}\right)$ can be computed from the VRP waypoint adjustment vector $\Delta \boldsymbol{v}_{w p}$ as

$$
\Delta \boldsymbol{\xi}_{a d j, \varphi}\left(t_{\varphi}\right)=\boldsymbol{A}_{\boldsymbol{\xi}_{\text {ref }}, \varphi}\left(t_{\varphi}\right) \Delta \boldsymbol{v}_{w p} .
$$

Inserting (29) combined with (32) into (34) yields

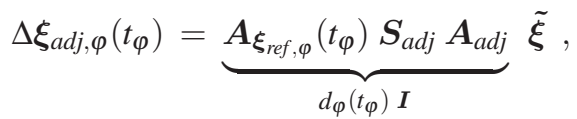

which shows how the DCM tracking error $\tilde{\xi}$ affects the current adjusted DCM reference trajectory $\Delta \boldsymbol{\xi}_{\text {adj, } \varphi}\left(t_{\varphi}\right)$. After each step adjustment, $d_{\varphi}\left(t_{\varphi}\right)$ starts from a value close to zero (i.e. $d_{\varphi}\left(t_{\varphi}\right) \ll 1$ ) and slowly grows until it reaches $d_{\varphi}\left(t_{\varphi}\right)=1$ at the beginning of the next step adjustment, i.e. for $\varphi=\varphi_{f a}$ and $t_{\varphi_{f a}}=0$, which is consistent with (31). This means that while (31) is only an assumption during the time before the step adjustment, it becomes true at the instant of step adjustment. In other words: while the adjusted DCM reference $\boldsymbol{\xi}_{a d j, \varphi}\left(t_{\varphi}\right)$ starts from a value very close to the nominal DCM reference $\boldsymbol{\xi}_{r e f, \varphi}\left(t_{\varphi}\right)$, it is continuously relaxed via (33) to be in perfect agreement with the current DCM (i.e. $\boldsymbol{\xi}_{a d j, \varphi}\left(t_{\varphi}\right)=\boldsymbol{\xi}$ ) at the instant of step adjustment. The main idea presented in this section is to make the DCM $\xi$ asymptotically track the adjusted DCM reference $\boldsymbol{\xi}_{\text {adj, } \varphi}\left(t_{\varphi}\right)$, i.e. we want to achieve

$$
\dot{\boldsymbol{\xi}}-\dot{\boldsymbol{\xi}}_{a d j, \varphi}\left(t_{\varphi}\right)=-\boldsymbol{K}_{\xi}\left(\boldsymbol{\xi}-\boldsymbol{\xi}_{a d j, \varphi}\left(t_{\varphi}\right)\right) .
$$

This is a stable first order dynamics if $\boldsymbol{K}_{\boldsymbol{\xi}}$ is positive definite. However, this asymptotic stability is deceptive, since $\boldsymbol{\xi}_{a d j, \varphi}\left(t_{\varphi}\right)$ depends on the DCM tracking error $\tilde{\boldsymbol{\xi}}$ (see (35)). We will thus examine the stability of the DCM $\boldsymbol{\xi}$ w.r.t. the original DCM reference $\boldsymbol{\xi}_{r e f, \varphi}\left(t_{\varphi}\right)$ in more detail. Using (3), we formulate the adjusted DCM reference velocity as

$$
\begin{aligned}
\dot{\boldsymbol{\xi}}_{a d j, \varphi}\left(t_{\varphi}\right) & =\frac{1}{b}(\boldsymbol{\xi}_{a d j, \varphi}\left(t_{\varphi}\right)-\underbrace{\boldsymbol{v}_{a d j, \varphi}\left(t_{\varphi}\right)}_{=\boldsymbol{v}_{r e f, \varphi}\left(t_{\varphi}\right)}) \\
& =\underbrace{\frac{1}{b}\left(\boldsymbol{\xi}_{r e f, \varphi}\left(t_{\varphi}\right)-\boldsymbol{v}_{r e f, \varphi}\left(t_{\varphi}\right)\right)}_{\dot{\boldsymbol{\xi}}_{r e f, \varphi}\left(t_{\varphi}\right)}+\frac{1}{b} \Delta \boldsymbol{\xi}_{a d j, \varphi}\left(t_{\varphi}\right)
\end{aligned}
$$

Here, the equality $\boldsymbol{v}_{a d j, \varphi}\left(t_{\varphi}\right)=\boldsymbol{v}_{r e f, \varphi}\left(t_{\varphi}\right)$ (upper underbrace) indicates that VRP reference trajectories for which $\varphi<\varphi_{f a}$ holds are not affected by the step adjustment.

With (33) and (37), equation (36) turns into

$$
\underbrace{\dot{\boldsymbol{\xi}}-\dot{\boldsymbol{\xi}}_{r e f, \varphi}\left(t_{\varphi}\right)}_{\tilde{\tilde{\boldsymbol{\xi}}}}=\frac{1}{b} \Delta \boldsymbol{\xi}_{a d j, \varphi}\left(t_{\varphi}\right)-\boldsymbol{K}_{\xi}\left(\tilde{\boldsymbol{\xi}}-\Delta \boldsymbol{\xi}_{a d j, \varphi}\left(t_{\varphi}\right)\right) \text {. }
$$

Inserting (35) yields the dynamics of the DCM error (remember: $\left.\tilde{\boldsymbol{\xi}}=\boldsymbol{\xi}-\boldsymbol{\xi}_{\text {ref, } \varphi}\left(t_{\varphi}\right)\right)$ as

$$
\dot{\tilde{\boldsymbol{\xi}}}=\underbrace{\left(\frac{d_{\varphi}\left(t_{\varphi}\right)}{b} \boldsymbol{I}-\boldsymbol{K}_{\xi}\left(1-d_{\varphi}\left(t_{\varphi}\right)\right)\right)}_{\boldsymbol{K}_{\xi, a d j}} \tilde{\boldsymbol{\xi}}
$$

This DCM dynamics is achieved by the following VRP based DCM controller (note the similarity to control law (24))

$$
\boldsymbol{v}=\boldsymbol{v}_{r e f, \varphi}\left(t_{\varphi}\right)+\left(1-d_{\varphi}\left(t_{\varphi}\right)\right)\left(\boldsymbol{I}+\boldsymbol{K}_{\xi} b\right) \tilde{\xi} .
$$

This control law uses the nominal VRP reference $\boldsymbol{v}_{r e f, \varphi}\left(t_{\varphi}\right)$ and the DCM tracking error $\tilde{\xi}$ as input to compute the desired VRP position $\boldsymbol{v}$. Inserting the latter into (4) returns the desired linear CoM force, which can be commanded to a higher level controller.

As described above, $d_{\varphi}\left(t_{\varphi}\right)$ tends from $\ll 1$ to 1 until the instant of step adjustment. This means that matrix $\boldsymbol{K}_{\xi, a d j}$ in (39) tends from $\approx-\boldsymbol{K}_{\xi} \boldsymbol{I}$ to $\frac{1}{b} \boldsymbol{I}$ accordingly, which reveals the behavior of the overall controller: At times long before the step adjustment it acts as a tracking controller, which helps compensate the DCM tracking error via force modulation, whereas the closer the instant of step adjustment approaches the more the DCM regains its natural divergent behavior (compare to (3)) and the more the DCM stabilization relies on the foreseen step adjustment.

This weakened tracking control performance of (40) as compared to (24) yields two major advantages: smoothness of the adjusted DCM reference trajectory and improved feasibility as compared to (24) (note: $\boldsymbol{v}$ tends to the VRP reference $\boldsymbol{v}_{r e f, \varphi}\left(t_{\varphi}\right)$, which is designed to be feasible, until the instant of step adjustment).

Inserting (3) and (37) into (36) yields an alternative formulation of (40):

$$
\boldsymbol{v}=\boldsymbol{v}_{r e f, \varphi}\left(t_{\varphi}\right)+\left(\boldsymbol{I}+\boldsymbol{K}_{\xi} b\right)\left(\boldsymbol{\xi}-\boldsymbol{\xi}_{a d j, \varphi}\left(t_{\varphi}\right)\right) .
$$

This controller is almost equivalent to (24), only that the original DCM reference $\boldsymbol{\xi}_{\text {ref, }, \varphi}\left(t_{\varphi}\right)$ is replaced by the adjusted DCM reference $\boldsymbol{\xi}_{a d j, \varphi}\left(t_{\varphi}\right)$.

\section{Integration of a momentum-based disturbance observer}

The method for DCM-based tracking control and step adjustment, presented in the previous sections, provides a framework for flexible, continuous and robust walking control. So far, the proposed controller has no knowledge (such as direction and magnitude) of external perturbations, which acts on the robot. Therefore, in case of strong and especially continuous perturbations, it may encounter actuation constraints (such as base of support) and struggle to recover. An estimate of the external perturbation force currently acting on the robot can increase the robustness of the controller drastically. Inspired by the work of DeLuca [21], Englsberger introduced a momentum-based disturbance observer in [18] that provides an estimate of the linear perturbation force acting on the robot. In the following, we will present the most important equations that define this disturbance observer. For further details, the reader is referred to [18]. The disturbance observer starts from the initial ${ }^{5}$ linear momentum $\boldsymbol{p}_{0}=m \dot{\boldsymbol{x}}(0)$ and integrates the known (commanded) force $\boldsymbol{F}_{\text {known }}(t)$ and the estimated

${ }^{5}$ when the disturbance observer is initialized 
perturbation force $\hat{\boldsymbol{F}}_{\text {perturb }}(t)$ to obtain the estimated linear momentum $\hat{\boldsymbol{p}}(t)$ at a given time $t$ :

$$
\hat{\boldsymbol{p}}(t)=\boldsymbol{p}_{0}+\int_{0}^{t}\left(\boldsymbol{F}_{\text {known }}(t)+\hat{\boldsymbol{F}}_{\text {perturb }}(t)\right) d t .
$$

As update law for the perturbation force estimate we use

$$
\hat{\boldsymbol{F}}_{\text {perturb }}(t)=k_{F}(\underbrace{m \dot{\boldsymbol{x}}(t)}_{\boldsymbol{p}(t)}-\hat{\boldsymbol{p}}(t)) .
$$

Here, $\boldsymbol{p}(t)$ denotes the actual linear momentum, which is either measured or provided by a state observer. The use of (42) and (43) yields following observer dynamics:

$$
\dot{\hat{\boldsymbol{F}}}_{\text {perturb }}(t)=k_{F}\left(\boldsymbol{F}_{\text {perturb }}(t)-\hat{\boldsymbol{F}}_{\text {perturb }}(t)\right),
$$

i.e. for $k_{F}>0$ the perturbation force estimate $\hat{\boldsymbol{F}}_{\text {perturb }}(t)$ follows the actual perturbation force $\boldsymbol{F}_{\text {perturb }}(t)$ with a stable first-order behavior ${ }^{6}$. For their application in discrete systems, equations (43) and (42) are discretized, which yields

$$
\hat{\boldsymbol{F}}_{\text {perturb }, k}=k_{F}\left(m \dot{\boldsymbol{x}}_{k}-\hat{\boldsymbol{p}}_{k}\right)
$$

and

$$
\hat{\boldsymbol{p}}_{k+1}=\hat{\boldsymbol{p}}_{k}+\left(\boldsymbol{F}_{\text {known }, k}+\hat{\boldsymbol{F}}_{\text {perturb }, k}\right) \Delta t,
$$

respectively. Here, $\Delta t$ denotes the sampling time of the discrete system. For $k=0, \hat{\boldsymbol{p}}_{k}$ is initialized as $\hat{\boldsymbol{p}}_{0}=m \dot{\boldsymbol{x}}(0)$.

In the presented work, the perturbation force estimate $\hat{\boldsymbol{F}}_{\text {perturb }, k}$ from (45) is used to shift all VRP and DCM reference quantities derived in the previous sections by an offset of $-\frac{b^{2}}{m} \hat{\boldsymbol{F}}_{\text {perturb }, k}$ (for further details on the corresponding derivation, refer to [18]). For the practical application, it is sufficient to simply replace all reference quantities in (24) or (41), respectively, by their shifted counterparts, i.e. by

$$
\boldsymbol{v}_{r e f, \varphi, s h i f t}\left(t_{\varphi}\right)=\boldsymbol{v}_{r e f, \varphi}\left(t_{\varphi}\right)-\frac{b^{2}}{m} \hat{\boldsymbol{F}}_{p e r t u r b, k}
$$

and

$$
\boldsymbol{\xi}_{\text {ref }, \varphi, \text { shift }}\left(t_{\varphi}\right)=\boldsymbol{\xi}_{\text {ref }, \varphi}\left(t_{\varphi}\right)-\frac{b^{2}}{m} \hat{\boldsymbol{F}}_{\text {perturb }, k}
$$

or

$$
\boldsymbol{\xi}_{a d j, \varphi, s h i f t}\left(t_{\varphi}\right)=\boldsymbol{\xi}_{a d j, \varphi}\left(t_{\varphi}\right)-\frac{b^{2}}{m} \hat{\boldsymbol{F}}_{\text {perturb }, k},
$$

respectively. Through this shift of reference quantities in the opposite direction of the perturbation force, the linear components of the perturbation force are directly compensated by the robot's end effector forces, while the torque that the perturbation force creates around the contact force reference point (eCMP, see [6], [18]) is compensated by gravity. The same "leaning" behavior is observed in humans, e.g. when playing tug of war. The overall dynamics achieved by the combined application of the described disturbance observer and the corresponding VRP and DCM reference shift was shown to be stable in [18].

\footnotetext{
${ }^{6}$ Note: in contrast to [17], our disturbance observer does not depend on the feedback of measured ground reaction forces.
}

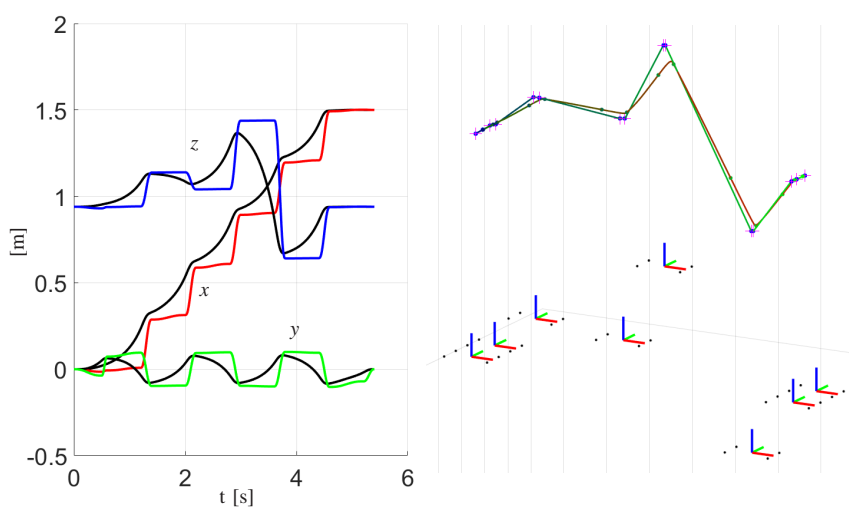

Fig. 1. Planning of smooth and consistent VRP and DCM references. a) black: DCM, colored: VRP, $\quad$ b) 3D visualization

\section{EVAluATION OF PRESENTED METHODS}

In the previous sections, we introduced methods for smooth and consistent gait generation and step adjustment. Figure 1 shows an example output of the gait generator: three-dimensional, smooth and consistent VRP and DCM trajectories, here for a standing to walking to standing transition. The coordinate frames denote foot centers. Time trajectories are plotted on the left, while spatial trajectories are shown on the right.

To evaluate the proposed push recovery methods, we performed numerous simulations of the humanoid robot Toro [22] in OpenHRP [23]. The design parameter $\Delta z_{v r p}$ was chosen to be $0.94 \mathrm{~m}$. Figure 2 presents the results of a step adjustment simulation, in which Toro was pushed by a lateral force ( $\pm 25 \mathrm{~N}$ for 1 second each) while walking forward on a rigid and flat floor. The plots on the left show the resulting measured and (adjusted) reference DCM trajectories. The adjusted DCM reference is smooth. After the perturbations, close to perfect DCM tracking is regained after a few steps.

The momentum-based disturbance observer presented in Sec. III-C was also tested in OpenHRP simulations. Considering the perturbation force estimate, the controller survives external forces of up to $15 \%$ of the robot's weight (ramping up from zero within e.g. 6 seconds).

\section{CONCLUSION AND FUTURE WORK}

In this paper, we presented a novel multi-step closedform walking trajectory generator based on the concept of Divergent Component of Motion (DCM). The resulting VRP and DCM trajectories are smooth and consistent. Also, we introduced an analytical method for step adjustment, which allows for the recovery of strong external perturbations. The DCM reference trajectory is smoothly adjusted. Finally, we introduced a momentum-based disturbance observer, which improves the robustness of the control framework w.r.t. strong and persisting perturbations. The methods were verified in numerous simulations. 


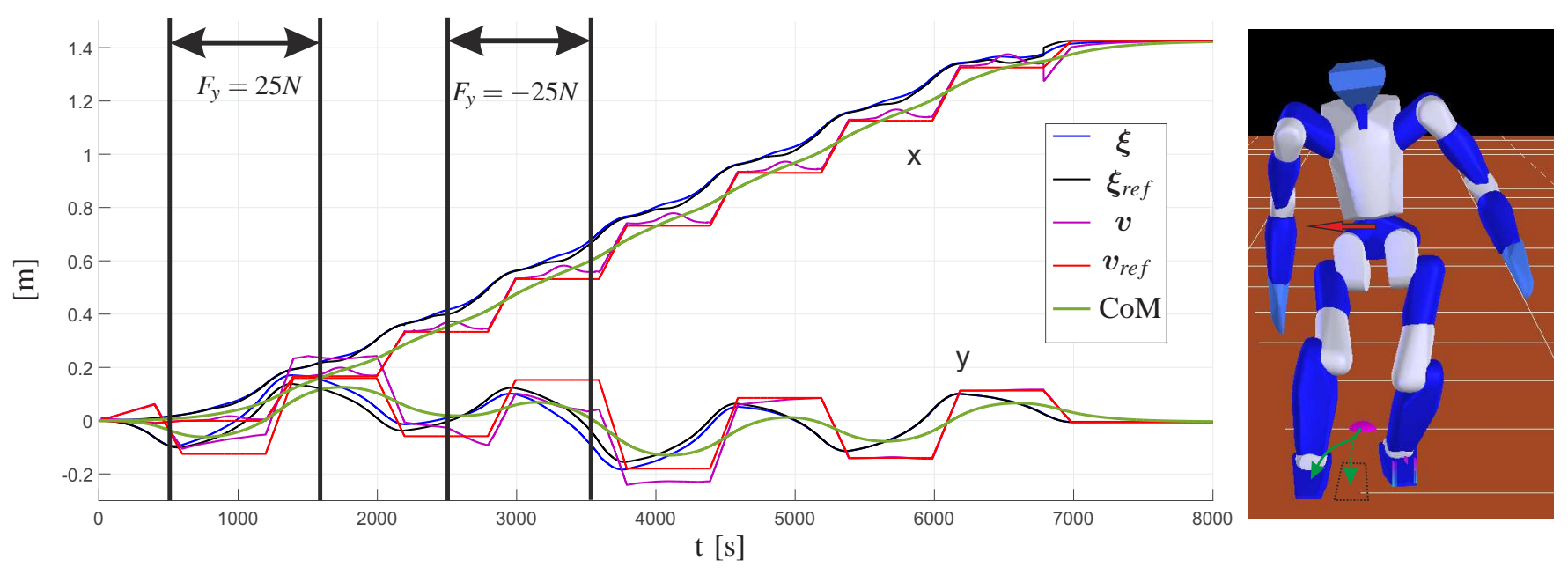

Fig. 2. Open HRP [23] simulation of humanoid robot Toro [22] subject to external perturbation forces.

In the future, we intend to extend our analytical derivations to include CoM reference trajectories and all controlled trajectories. These analytical previews will be helpful for the design and analysis of constraint-compatible trajectories. Also, we plan to evaluate the presented methods experimentally on our humanoid robot Toro [22]. Finally, we plan to extend the presented analytical methods to a corresponding MPC framework to explicitly consider actuation constraints.

\section{ACKNOWLEDGEMENTS}

This work was partially supported by the European Commission (H2020-ICT-645097 COMANOID).

\section{REFERENCES}

[1] S. Kajita, F. Kanehiro, K. Kaneko, K. Yokoi, and H. Hirukawa, "The $3 \mathrm{~d}$ linear inverted pendulum mode: A simple modeling for a biped walking pattern generation," in IEEE Int. Conf. on Robotics and Automation, 2001, pp. 239-246.

[2] R. Tedrake, S. Kuindersma, R. Deits, and K. Miura, "A closed-form solution for real-time zmp gait generation and feedback stabilization," in Humanoid Robots (Humanoids), 2015 IEEE-RAS 15th International Conference on, 2015, pp. 936-940.

[3] K. Harada, S. Kajita, K. Kaneko, and H. Hirukawa, "An analytical method on real-time gait planning for a humanoid robot," in IEEERAS Int. Conf. on Humanoid Robots, 2004, pp. 640-655 Vol. 2.

[4] P.-B. Wieber, "Trajectory free linear model predictive control for stable walking in the presence of strong perturbations," in IEEE-RAS Int. Conf. on Humanoid Robots, 2006, pp. 137-142.

[5] T. Takenaka, T. Matsumoto, and T. Yoshiike, "Real time motion generation and control for biped robot, 1st report: Walking gait pattern generation," in Int. Conf. on Intell. Robots and Systems, 2009.

[6] J. Englsberger, C. Ott, and A. Albu-Schäffer, "Three-dimensional bipedal walking control based on divergent component of motion," Robotics, IEEE Transactions on, vol. 31, no. 2, pp. 355-368, 2015.

[7] T. Koolen, T. D. Boer, J. Rebula, A. Goswami, and J. E. Pratt, "Capturability-based analysis and control of legged locomotion. part 1: Theory and application to three simple gait models," Int. J. of Robotics Research, vol. 31, no. 9, pp. 1094-1113, 2012.

[8] M. A. Hopkins, D. W. Hong, and A. Leonessa, "Humanoid locomotion on uneven terrain using the time-varying divergent component of motion," in 2014 IEEE-RAS International Conference on Humanoid Robots, 2014, pp. 266-272.

[9] $\longrightarrow$, "Compliant locomotion using whole-body control and divergent component of motion tracking," in 2015 IEEE International Conference on Robotics and Automation (ICRA), 2015, pp. 5726-5733.
[10] S. k. Yun and A. Goswami, "Momentum-based reactive stepping controller on level and non-level ground for humanoid robot push recovery," in 2011 IEEE/RSJ International Conference on Intelligent Robots and Systems, 2011, pp. 3943-3950.

[11] J. Urata, K. Nishiwaki, Y. Nakanishi, K. Okada, S. Kagami, and M. Inaba, "Online decision of foot placement using singular LQ preview regulation." in IEEE-RAS Int. Conf. on Humanoid Robots, 2011.

[12] H. Diedam, D. Dimitrov, P.-B. Wieber, K. Mombaur, and M. Diehl, "Online walking gait generation with adaptive foot positioning through linear model predictive control," in Int. Conf. on Intell. Robots and Systems, 2008, pp. 1121-1126.

[13] M. Khadiv, A. Herzog, S. A. A. Moosavian, and L. Righetti, "Step timing adjustment: A step toward generating robust gaits," in Humanoid Robots (Humanoids), 2016 IEEE-RAS 16th International Conference on. IEEE, 2016, pp. 35-42.

[14] M. Khadiv, S. Kleff, A. Herzog, S. A. Moosavian, S. Schaal, L. Righetti et al., "Stepping stabilization using a combination of dcm tracking and step adjustment," arXiv preprint arXiv:1609.09822, 2016.

[15] B. Stephens, "Humanoid push recovery," in Humanoid Robots, 2007 7th IEEE-RAS International Conference on, 2007, pp. 589-595.

[16] B. Stephens and C. Atkeson, "Push recovery by stepping for humanoid robots with force controlled joints," in IEEE-RAS Int. Conf. on Humanoid Robots, 2010, pp. 52-59.

[17] R. J. Griffin, A. Leonessa, and A. Asbeck, "Disturbance compensation and step optimization for push recovery," in 2016 IEEE/RSJ International Conference on Intelligent Robots and Systems (IROS), 2016, pp. 5385-5390.

[18] J. Englsberger, "Combining reduced dynamics models and wholebody control for agile humanoid locomotion," Dissertation, Technische Universität München, München, 2016.

[19] G. Mesesan, J. Englsberger, B. Henze, and C. Ott, "Dynamic multicontact transitions for humanoid robots using divergent component of motion," in IEEE Int. Conf. on Robotics and Automation, 2017.

[20] J. Englsberger, C. Ott, and A. Albu-Schäffer, "Three-dimensional bipedal walking control using divergent component of motion," in Int. Conf. on Intell. Robots and Systems, 2013, pp. 2600-2607.

[21] A. De Luca, A. Albu-Schäffer, S. Haddadin, and G. Hirzinger, "Collision detection and safe reaction with the dlr-iii lightweight manipulator arm," in Int. Conf. on Intell. Robots and Systems, 2006, pp. 1623-1630.

[22] J. Englsberger, A. Werner, C. Ott, B. Henze, M. A. Roa, G. Garofalo, R. Burger, A. Beyer, O. Eiberger, K. Schmid, and A. Albu-Schäffer, "Overview of the torque-controlled humanoid robot toro," in IEEERAS Int. Conf. on Humanoid Robots, 2014, pp. 916-923.

[23] F. Kanehiro, K. Fujiwara, S. Kajita, K. Yokoi, K. Kaneko, H. Hirukawa, Y. Nakamura, and K. Yamane, "Open architecture humanoid robotics platform," in IEEE Int. Conf. on Robotics and Automation, 2002, pp. 24-30 vol.1. 